\title{
Bang-bang decoupling used in quantum optics for the first time
}

Sajeev Damodarakurup, Marco Lucamarini, Giovanni Di Giuseppe, David Vitali, and Paolo Tombesi

Experimental suppression of polarization decoherence in a ring cavity uses a simple open-loop control technique known as bang-bang decoupling.

In recent years new strategies for maintaining the coherence (i.e., constant relative phase) of quantum systems have appeared. ${ }^{1,2}$ Even if photons interact weakly with their surroundings, the resulting decoherence (i.e., randomization of the relative phase) may significantly affect their polarization state during propagation within dispersive media. We show how the polarization decoherence of a photon circling in a ring cavity can be suppressed by adapting to the field of quantum optics the open-loop protection technique known as bang-bang (BB). This method is already being employed for nuclear spins and nuclear-quadrupole qubits. It has potential practical application in the field of optical fibers.

In $\mathrm{BB}$, the system undergoes a sequence of suitably tailored unitary operations aimed at decoupling the system from the surrounding environment. For a polarization qubit, conveniently represented as a point on the surface of a Bloch sphere (see Figure 1), BB consists of alternating fast $180^{\circ}$ flips of the polarization state about the two axes, $z$ and $x .^{3}$ The physical idea behind BB comes from nuclear magnetic resonance (NMR) spectroscopy, where the controls are implemented in time as a sequence of strong and rapid pulses. In the photon case BB is applied in space: while circling in the cavity, the photon passes directly through the optical elements that implement the BB transformations on it. Figure 2 depicts our experimental apparatus. A laser diode at $800 \mathrm{~nm}$ with a bandwidth of about $15 \mathrm{~nm}$ is pulsed at $100 \mathrm{KHz}$. The laser is injected into a triangular ring cavity composed of a spherical mirror and two planar mirrors, and the beam is attenuated so that it provides an average of one photon per pulse.

In the cavity the photon undergoes two kinds of polarization decoherence. One arises from the polarization-dependent reflectivity of the plane mirrors. We introduce the other manually: it

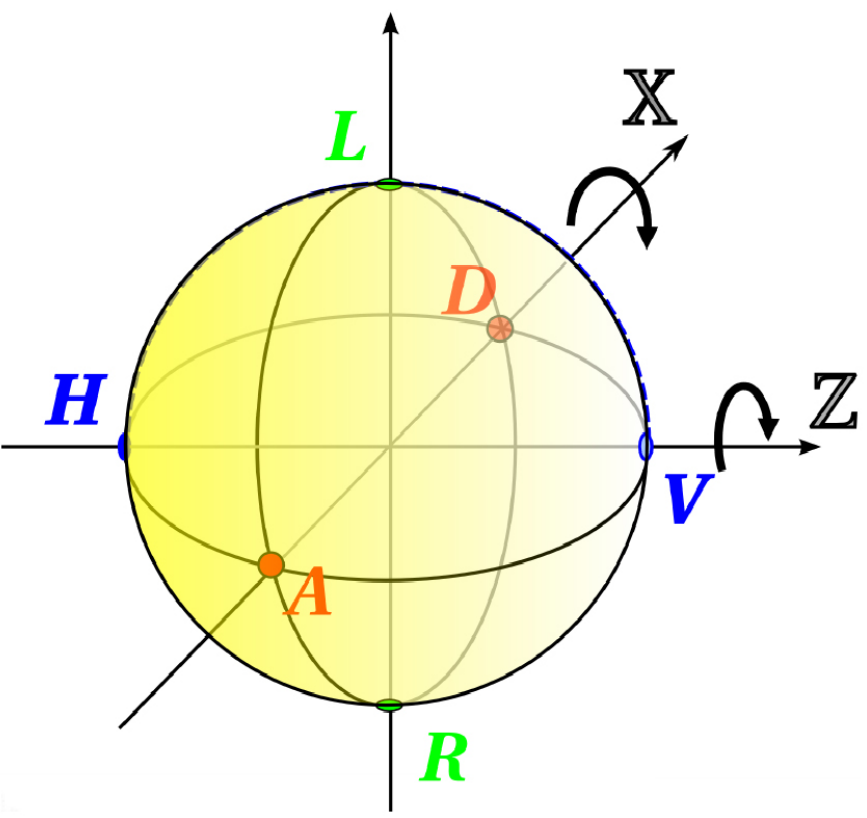

Figure 1. Bloch-sphere representation of polarization states of light. The $B B$ unitary operations, $\mathbb{X}$ and $\mathbb{Z}$, correspond to $\pi$-rotations about the $x$ - and z-axes. $\{H, V\},\{D, A\}$, and $\{R, L\}$ represent the eigenstates of the three polarization bases.

comes from the birefringent crystals positioned in front of the plane mirrors, which are labeled $B_{X}$ in Figure 2. These crystals introduce a controlled birefringence along various axes of the Bloch sphere. We quantify the amount of decoherence by evaluating the purity of the experimentally reconstructed output density matrices (see Figure 3). A full BB decoupling cycle is composed of the double application of the $\mathbb{X}$ and $\mathbb{Z}$ flips during the system evolution. Two waveplates, labeled $\mathrm{X}$ and $\mathrm{Z}$ in Figure 2, make these flips occur.

In our first experiment we studied the mirror-reflectivitydependent decoherence for different polarization input states. Figure 3 reports the corresponding output density 


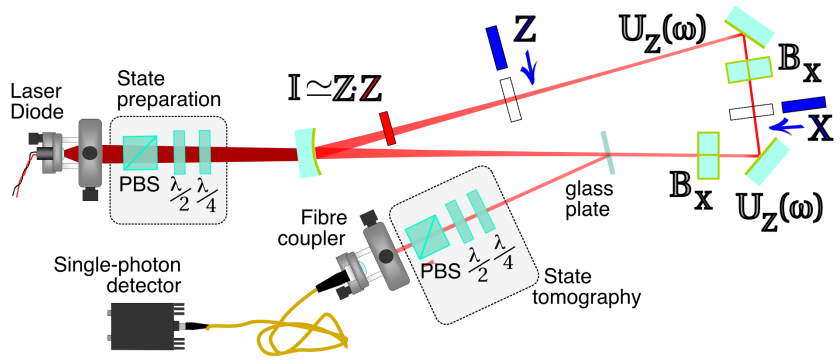

Figure 2. Schematics of our experiment. The preparation box is composed of a polarizing beam splitter (PBS) and a combination of $\lambda / 4$ and $\lambda / 2$ waveplates. Analogously, the tomographic measurement box is composed of a pair of motorized $\lambda / 2$ and $\lambda / 4$ waveplates and a PBS, followed by a single-photon detector. $\mathbb{I} \simeq \mathbb{Z} \cdot \mathbb{Z}$ is the identity operator, while $\mathbb{U}_{\mathbb{Z}}(\omega)$ is the mirror operator causing decoherence. $B_{X}$ : Birefringent crystal.
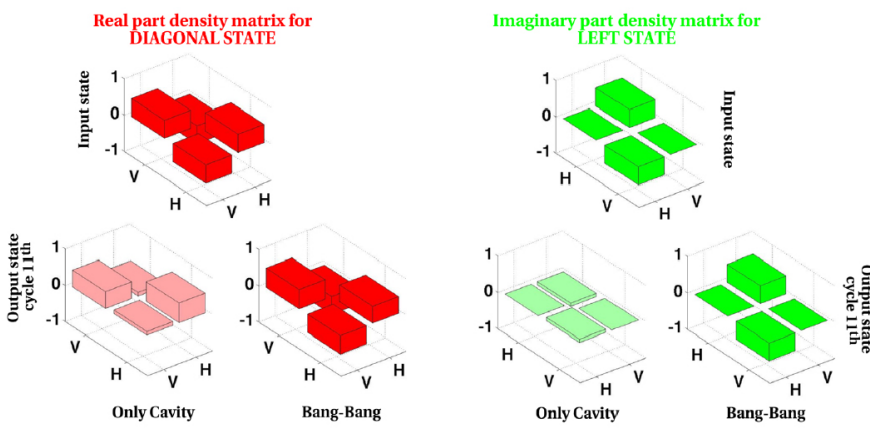

Figure 3. (left) The real part of the density matrix of the input state $D$ (red, on the top), of the output state when BB is not applied (light red), and of the output state when 11 BB cycles are applied (dark red). (right) The imaginary part of the density matrix of the input state $L$ (green, on the top), of the output state without BB (light green), and of the output state with 11 BB cycles (dark green).

matrices. Since reflection at plane mirrors leaves horizontal and vertical polarizations unaltered, the cavity does not introduce any decoherence for these states. However, the situation is different for diagonal and circular polarization states, as they fall into a completely mixed state after a photon travels around the cavity a few times in the absence of the BB gates. This can be seen in Figure 3 from the density matrix of diagonal elements, which average to zero in the absence of BB. On the contrary, after the introduction of $\mathrm{BB}$, the purity of the qubits is nearly identical to its maximum value, even after several cavity round trips, as shown by the presence of nonzero density matrix of diagonal elements.

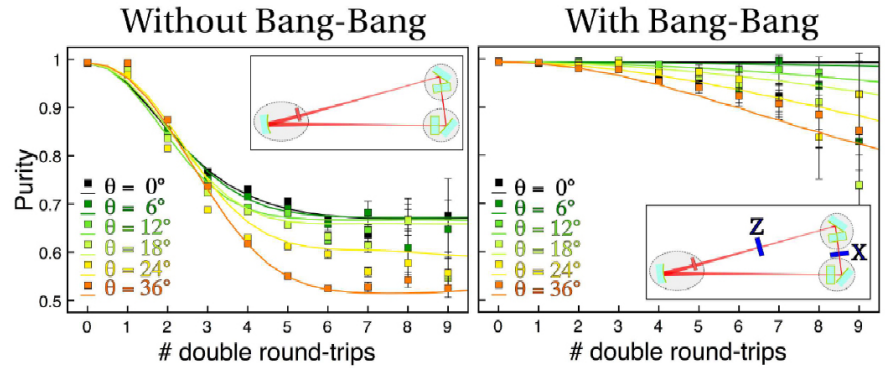

Figure 4. The experimental results show that BB controls for birefringence along a general axis. The input polarization is elliptical.

To test BB in a more general scenario, we performed a second experiment using birefringent crystals $B_{X}$. In case of no $\mathrm{BB}$, the resulting fast polarization decay is shown in the left side of Figure 4 for a general elliptical polarization state, for different alignment directions of the crystals. The recovery of purity after the application of $\mathrm{BB}$ is apparent from the right side of the figure, and proves the usefulness of the scheme to combat a general birefringence-induced decoherence.

In conclusion, we have conducted the first experimental application of BB open-loop decoupling in the field of quantum optics. Specifically, we applied BB control to the recovery of birefringence-induced polarization decoherence in a triangular ring cavity. We verified that BB dramatically reduced the decoherence in terms of the purity of the output states. Although not reported here, we also verified the effectiveness of $\mathrm{BB}$ in increasing the overall fidelity of photon transmission through the cavity. In future work we will develop a direct experimental demonstration of this effect in a one-way single-mode optical fiber.

\section{Author Information}

Sajeev Damodarakurup, Marco Lucamarini, Giovanni Di Giuseppe, David Vitali, and Paolo Tombesi Department of Physics

University of Camerino

Camerino, Italy

http:/ / camcat.df.unicam.it/?q=node/10

http:/ /camcat.df.unicam.it/

Sajeev Damodarakurup is a $\mathrm{PhD}$ fellow within the European Union (EU)-funded Qubit Applications (QAP) project in the Department of Physics at the University of Camerino, 
Italy. His scientific research deals with experimental photonics at the quantum level, quantum information, and quantum decoherence.

Marco Lucamarini is a postdoctoral fellow within the EU-funded QAP project in the Department of Physics at the University of Camerino. His interests include both theoretical and experimental quantum cryptography and decoherence, mainly within the field of quantum optics. Lucamarini has co-authored two articles for SPIE.

Giovanni Di Giuseppe is a researcher. He has worked on nonlinear optics, ultrafast optical phenomena, quantum state tomography, entanglement engineering, and recently on quantum information and cryptography. He is a contributor to Italian- and European-funded projects. He has co-authored two articles for SPIE.

David Vitali is a professor of the physics of matter and coordinator of the physics master's programs at the University of Camerino. His research focuses on quantum optics and quantum information, and he has participated in and coordinated both national and European projects in this research area.
Paolo Tombesi is a professor in and chair of the Department of Physics at the University of Camerino. His research activities include quantum optics and quantum information. He is a member of the Optical Society of America, the Societa Italianà di Fisica, European Physical Society, American Physical Society, and American Association for the Advancement of Science, and a contributor to several Italian- and European-funded projects. He has co-authored two papers for SPIE.

\section{References}

1. L. Viola and S. Lloyd, Dynamical suppression of decoherence in two-state quantum systems, Phys. Rev. A 58, p. 2733, 1998.

2. D. Vitali and P. Tombesi, Using parity kicks for decoherence control, Phys. Rev. A 59, p. 4178,1999 .

3. S. Massar and S. Popescu, Reducing polarization mode dispersion with controlled polarization rotations, New J. Phys. 9, p. 158, 2007. 